\title{
Image Enhancement through Denoising and Retrieval of Vegetation Parameters from Landsat8
}

\author{
K. Sateesh Kumar ${ }^{\#}$, G.Sreenivasulu ${ }^{\#}$ \\ ${ }^{\#}$ Department of Electronics and Communication Engineering, \\ Sri Venkateswara University College of Engineering, S V University, Tirupati, AndhraPradesh-517502, India \\ E-mail : sateeshkumarkanagala@gmail.com
}

\begin{abstract}
This paper proposed the enhancement of Landsat8 imagery through an Un-decimated Dual-Tree Complex Wavelet Transform (UDT-CWT) based denoising method and modified homographic filter for edge preservation. This work has been extended by estimating several vegetation parameters like Normalized Difference of Vegetation Index (NDVI), Green Normalized Difference Vegetation Index (GNDVI), Modified Soil Adjusted Vegetation Index (MASVI), and Soil \& Atmospherically Resistant Vegetation Index (SARVI). Once the estimation of these parameters was done, the effect of noise was verified. Wavelet decomposes the image into frequency subbands and de-noises each subband separately. These subbands help to increase the resolution. The general problem of the homomorphic filter is that it doesn't enhance the Low-frequency components which also play a key role in estimating Vegetation Indices (VI).So it was modified to enhance the high-frequency components as well as low-frequency details. Monitoring of vegetation parameters using remote sensing is one of the prominent ways in the estimation of crop yield, Land Use Land Cover (LULC), Water resource management, Drought management, etc. The high-resolution image is more preferable than moderate resolution image to retrieve VI. Image denoising and enhancing the spatial resolution helps to retrieve the parameters well and accurate. The proposed algorithm was working on the images of Landsat8.
\end{abstract}

Keywords - Homographic filter; Landsat8; Un-decimated Dual-Tree Complex Wavelet Transform; Vegetation Indices.

\section{INTRODUCTION}

The Discrete Wavelet Transform (DWT) has been effectively used in image processing in the area of denoising, fusion, and compression, etc. The 2D Wavelet-based denoising method [1]-[5] divides the image into approximate bands (LL), vertical band (LH), horizontal (HL) and diagonal $(\mathrm{HH})$ by row wise and column wise successively liable to cover the entire spectrum of the original image. The lower subband edges support to estimate the edges of higher subband on the basis of wavelet coefficients.

DT-CWT decomposition frames two parallel trees (real and imaginary). Each tree has a specific filter design for directional effective selectivity. It suffers from phase variations which are focused in this paper using Undecimated Complex Wavelet Transform (UDT-CWT). It eliminates the concept of down-sampling, which is present in DWT [6]. Enhancement of resolution is one of the growing fields in remote sensing [7]-[9]. The homomorphic filter was used for enhancement of satellite imagery. Actually, the enhancement discussed for the radiographic images [10] was extended to remote sensing images. This paper is framed by adding Zero means Additive White Gaussian Noise (AWGN) for denoising. This method allows achieving the significant quality of the denoised image.
Vegetation indices based prediction of crop yield from remotely sensed imagery is trending in the remote sensing field. The real-time and historical weather data combined model predict the rice yield based on crop growth [11]-[13]. These indices place a vital role in deciding whether the year is a drought or not [14]. Remote sensing based Vegetation indices reflect the different phenology stages of crops [15]. These indices are like parameters for scaling plant growth and progress. Some of the vegetation indices (VI) account the land type too, and some of them are not [16]. Most of the considered vegetation indices are taking the reflectance of Near Infrared Band (NIR), Red band(R) and Blue (B) on the basis of their spectral properties. For better analysis VI are normalized. So, that the values spread over the range $(-1,1)$, where 1 remarks good vegetation (Forest) and -1 represents the water. Noisy Images always mislead retrieved parameters like NDVI, GNDVI, SARVI, and SAVI, etc. Geographically, Chittoor region is considered for the study \{78 $30^{\circ}-79^{\circ} 55^{\prime}$ E Longitude, $12^{\circ} 37^{\prime}-14^{\circ} 8^{\prime}$ N Latitude $\}$, since it is semi-arid region suitable for paddy, groundnut, sugarcane, etc. This region suffers from lack of sufficient water resources. So, most of the farmers prefer dry crops rather than wet crops. The whole crop year is divided into two seasons, i.e., Kharif and Rabi. The whole study has been taken from 2015-2017 on the seasonal basis. 


\section{MATERIALS AND METHOD}

\section{A. Data Collection}

Data used in this paper is OLI (Operational Land Imager) Sensor's Landsat 8 Imagery for Chittoor region downloaded from official website of United States Geological Survey (USGS) https://www.usgs.gov/. Table 1 represents all band designations with wavelength and resolution details.

TABLE I

BAND DESIGNATIONS IN LANDSAT8 SATELLITE

\begin{tabular}{|c|l|c|c|}
\hline No. & \multicolumn{1}{|c|}{ Bands } & $\begin{array}{c}\text { Wavelength } \\
\text { (mm.) }\end{array}$ & $\begin{array}{c}\text { Resolution } \\
\text { (meters.) }\end{array}$ \\
\hline 1. & CoastalAerosol & $0.43-0.45$ & 30 \\
\hline 2. & Blue & $0.45-0.51$ & \\
\hline 3. & Green & $0.53-0.59$ & 30 \\
\hline 4. & Red & $0.64-0.67$ & 30 \\
\hline 5. & Near Infrared & $0.85-0.88$ & 30 \\
\hline 6. & SWIR1 & $1.57-1.65$ & 30 \\
\hline 7. & SWIR2 & $2.11-2.29$ & 30 \\
\hline 8. & Panchromatic & $0.50-0.68$ & 15 \\
\hline 9. & Cirrus & $1.36-1.38$ & 30 \\
\hline 10. & Thermal Infrared 1 & $10.60-11.19$ & 30 \\
\hline 11. & Thermal Infrared 2 & $11.50-12.51$ & 30 \\
\hline
\end{tabular}

\section{B. Image Denoising}

Remote sensing imagery is not like a normal photograph. Clearly, a lot of features are being recorded in the form of radiance or reflectance. So, while denoising this type of imagery, utmost care should be taken. The proposed method consists of three stages; they are Denoising, Enhancement and finally retrieval of Vegetation parameters. For the DWT, a small change in an image results in large variation in the coefficients, aliasing effect and mediocre directional selectivity (DWT based denoising) [4]. To overcome this problem in remote sensed image denoising UDT-CWT was proposed. UDT-CWT has limited set of coefficients related to each subband and maintains the resolution of the image. The analysis of UDT-CWT is shown in Fig. 1. The main difference between DWT and UDT-CWT is avoiding downsampling. The undecimated wavelet filter at $\mathrm{l}+1$ sample is given by

$\mathrm{g}^{(\mathrm{l}+1)}[\mathrm{k}]=\mathrm{g}^{(\mathrm{l})}[\mathrm{k}] \uparrow 2=\mathrm{g}^{(\mathrm{l})}\left[\frac{\mathrm{k}}{2}\right]$, if $\mathrm{k}$ is even;0 Otherwise (1)

where is the non upsampled q-step filter.

It maintains the Excellency in denoising of DT-CWT in addition that it preserves the same resolution of each band. The proposed algorithm is one to one matching between coefficients in all subbands of Landsat8.Basically, this structure contains two similar biorthogonal filter banks (h0o, $\mathrm{h} 01)$ and $(\mathrm{g} 0 \mathrm{o}, \mathrm{g} 01)$ in which entire spectrum is divided into real and imaginary parts.

- Specially designed real filters for all first level.

- Dual filters for all remaining levels of DT-CWT.

At the stage of inverse UD-CWT, the upsampled filter was used in the process of reconstruction. The noisy image is decomposed into subbands using four-level DT-CWT with coefficients $+75^{\circ},+45^{\circ},+15^{\circ},-75^{\circ}$ and $-45^{\circ},-15^{\circ}$.
The analytic functions of the filters are to perform DTCWT wavelet analysis. It uses two real and two imaginary DWT trees. Mother wavelet $\Psi(\mathrm{t})$ and scaling function $\emptyset(\mathrm{t})$ are represented in the following Eq. (1) to Eq. (4)

$$
\begin{aligned}
\Psi_{\mathrm{r}}(\mathrm{t}) & =\sqrt{ } 2 \sum_{\mathrm{n}} \mathrm{H}_{\mathrm{a}}(\mathrm{n}) \emptyset_{\mathrm{r}}(2 \mathrm{t}-\mathrm{n}) \\
\Psi_{\mathrm{i}}(\mathrm{t}) & =\sqrt{ } 2 \sum_{\mathrm{n}} \mathrm{H}_{\mathrm{b}}(\mathrm{n}) \emptyset_{\mathrm{i}}(2 \mathrm{t}-\mathrm{n}) \\
\emptyset_{\mathrm{r}}(\mathrm{t}) & =\sqrt{ } 2 \sum_{\mathrm{n}} \mathrm{L}_{\mathrm{a}}(\mathrm{n}) \Psi_{\mathrm{r}}(2 \mathrm{t}-\mathrm{n}) \\
\emptyset_{\mathrm{i}}(\mathrm{t}) & =\sqrt{ } 2 \sum_{\mathrm{n}} \mathrm{L}_{\mathrm{b}}(\mathrm{n}) \Psi_{\mathrm{i}}(2 \mathrm{t}-\mathrm{n})
\end{aligned}
$$

Where $\mathrm{L}$ represents Low pass filters, and $\mathrm{H}$ represents high pass Filters. $\Psi_{r}, \Psi_{\mathrm{i}}$ Wavelet functions represent Complex Wavelet $\Psi_{\mathrm{c}} . \emptyset_{\mathrm{r}}, \emptyset_{\mathrm{i}}$ Wavelet functions represent Complex Wavelet Øc. The main reason for DT-CWT is shift invariant. It is able to discriminate positive as well as negative subbands.

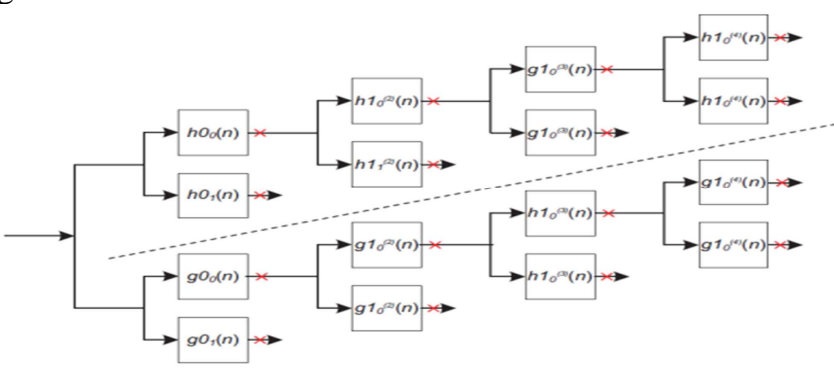

Fig. 1 Analysis stages of UDT-CWT. The crosses indicate the positions where down sampling would normally occur within the decimated DTCWT

The real and imaginary components of first stage DTCWT of output y is given in Eq. (5) to Eq. (7).

$$
\begin{gathered}
\mathrm{y}_{\mathrm{r}}[\mathrm{n}]=\sum_{\mathrm{k}} \mathrm{g}[\mathrm{k}] X[2 \mathrm{n}-\mathrm{k}] \\
\mathrm{y}_{\mathrm{i}}[\mathrm{n}]=\sum_{\mathrm{k}} \mathrm{g}[\mathrm{k}] X[2 \mathrm{n}-\mathrm{k}+1] \\
|\mathrm{y}[\mathrm{n}]|^{2}=\left|\mathrm{y}_{\mathrm{r}}[\mathrm{n}]\right|^{2}+\mathrm{i}\left|\mathrm{y}_{\mathrm{i}}[\mathrm{n}]\right|^{2}=\left(\mathrm{y}_{\mathrm{r}}[\mathrm{n}]\right)^{2}+\left(\mathrm{y}_{\mathrm{i}}[\mathrm{n}]\right)^{2}
\end{gathered}
$$

Where, $y[n]$ represents nth sample of the output, which is a combined form of $y_{r}[n], y_{i}[n]$.

The Output of this stage is a denoised version of the input image, and the quality of the image can be analyed using performance characteristics like Peak Signal to Noise Ratio (PSNR), Structural Similarity Index (SSIM), and Standard Deviation (SD).

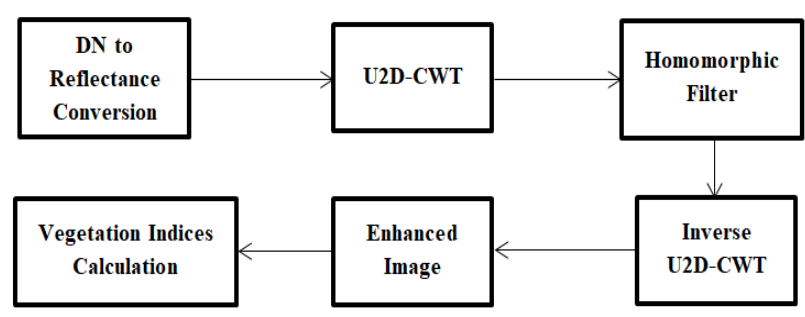

Fig. 2 Block diagram of proposed methodology

The block diagram of the proposed methodology is shown in Fig. 2. First, the DN values are converted into Top of Atmosphere (ToA) reflectance. The reflectance is corrected using a solar elevation angle. The corrected reflectance values are processed using Undecimated Dual Tree Complex 
Wavelet Transform (UDT-CWT). At this stage, the coefficients are enhanced with a modified homomorphic filter. To get back index values from coefficients, inverse U2D-CWT was used. VI was calculated using this enhanced image.

\section{Image Enhancement}

1) Homomorphic Filtering: The homomorphic filtering is for the enhancement of an image based on illumination and reflection model [18]. This helps to retain Highfrequency components. It deals with reflection and illumination separately.

$$
\mathrm{T}(\mathrm{u}, \mathrm{v})=\mathrm{F}_{\mathrm{r}}(\mathrm{u}, \mathrm{v})+\mathrm{F}_{\mathrm{l}}(\mathrm{u}, \mathrm{v})
$$

where $\mathrm{T}(\mathrm{u}, \mathrm{v})=$ High-frequency components after filtering $\mathrm{F}_{\mathrm{r}}(\mathrm{u}, \mathrm{v})$ and $\mathrm{F}_{\mathrm{l}}(\mathrm{u}, \mathrm{v})$ are Fourier transforms of illumination and reflection components. Output is filtered through

$$
S(u, v)=H(u, v) . T(u, v)
$$

By applying Inverse Fourier Transform to the filtered output (6) gives,

$$
\mathrm{s}(\mathrm{x}, \mathrm{y})=\mathrm{F}^{-1}\{\mathrm{~S}(\mathrm{u}, \mathrm{v})\}
$$

The high pass filter used in this is Butterworth filter

$$
H(u, v)=\frac{1}{1+\left[\frac{D_{0}}{D(u, v)}\right]^{2 n}}
$$

where $n$ represents the order of the filter, $D_{0}$ is the cut-off distance from the center and $\mathrm{D}(\mathrm{u}, \mathrm{v})$ is given by:

$$
\mathrm{D}(\mathrm{u}, \mathrm{v})=\left[(\mathrm{u}-\mathrm{M} / 2)^{2}+(\mathrm{v}-\mathrm{N} / 2)^{2}\right]^{1 / 2}
$$

where $\mathrm{M}$ and $\mathrm{N}$ represents the number of rows and columns

The Modified Homomorphic Filtering is a filter that improves high frequency, as well as the low frequency of the Fourier Transform, was designed.

$$
\mathrm{F}=\alpha * \mathrm{H}_{\mathrm{LPF}}+\beta * \mathrm{H}_{\mathrm{HPF}}
$$

where $\mathrm{H}_{\mathrm{LPF}}, \mathrm{H}_{\mathrm{HPF}}$ are related the Butterworth filter in Eq. (8) The values of $\alpha, \beta$ are satisfied the condition $\beta>\alpha \geq 0$.

The reason for adding low pass filter is to preserve lowfrequency features like water bodies, shrubs and crop details, etc. These details also have a vital role in deciding Vegetation indices. So, $\beta$ is chosen greater than 1 and $\alpha$ are less than 1.The below Fig. 3 describes the operation of the modified homomorphic filter. The value of $\alpha$ can be varied from 0 to 1 (preferably, $\alpha=0.8$ ) and Value of $\beta$ varied from 3 to 5 .

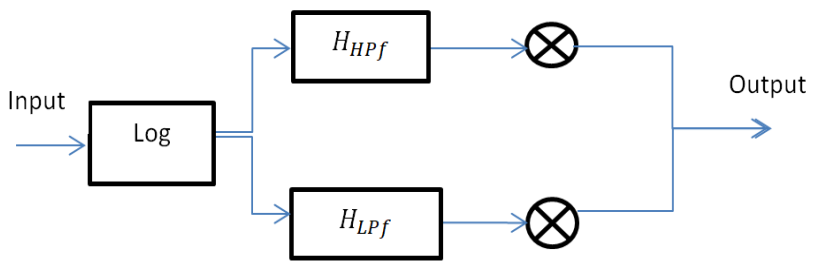

Fig. 3 Structure of modified homomorphic filter
This enhanced homomorphic filter is used in this paper to enhance the denoised satellite image. The denoised image is first converted to HSV plane. The V component is applied to modified Homomorphic filter and doesn't change H \& S planes. This modified V plane is added to $\mathrm{H}, \mathrm{S}$ planes which are further converted back to RGB plane. This RGB plane restores the colour information of the original image received. The Colour correction was also done using Gamma Correction technique.

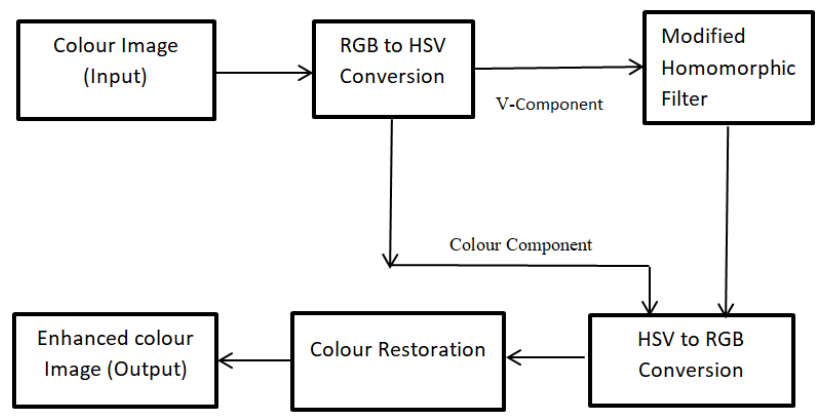

Fig. 4 Colour enhancement model for satellite imagery

\section{Vegetation Indices (VI)}

The following Vegetation Indices can be calculated using Landsat Data Spectral bands.

1) Normalized Difference Vegetation Index (NDVI): It is the ratio of Reflectance from NIR and Red bands. Its value is in the range of -1 to +1 , where +1 represents maximum vegetation. -1 relates to snow.

$$
\mathrm{NDVI}=\frac{\left(\rho_{\mathrm{NIR}}-\rho_{\mathrm{Red}}\right)}{\left(\rho_{\mathrm{NIR}}+\rho_{\mathrm{Red}}\right)}[15]
$$

2) Soil Adjusted Vegetation Index (SAVI): It is the scaled version of NIR and Red bands reflectance ratio.

$$
\mathrm{SAVI}=\frac{\left(\rho_{\mathrm{NIR}}-\rho_{\mathrm{Red}}\right)}{\left(\rho_{\mathrm{NIR}}+\rho_{\mathrm{Red}}\right)} *(1+\mathrm{L})[15]
$$

The value of $\mathrm{L}$ can be calculated as follows

$$
\mathrm{L}=1-\frac{2 * \mathrm{~S} *\left(\rho_{\mathrm{NIR}}-\rho_{\mathrm{Red}}\right) *\left(\rho_{\mathrm{NIR}}-\mathrm{S} * \rho_{\mathrm{Red}}\right)}{\left(\rho_{\mathrm{NIR}}-\rho_{\mathrm{Red}}\right)}[15]
$$

where $\mathrm{S}$ is the slope line of soil in the plot of NIR and Red brightness. In general, $\mathrm{L}$ is treated as 0.5 .

3) Modified Soil Adjusted Vegetation Index (MSAVI): It is the ratio of reflectance of NIR band and Red band with some correction factor $\mathrm{L}$.

$$
\operatorname{MSAVI}=\left(\rho_{\mathrm{NIR}}-\rho_{\mathrm{Red}}\right)(1+\mathrm{L}) /\left(\rho_{\mathrm{NIR}}+\rho_{\mathrm{Red}}+\mathrm{L}\right)[15]
$$

The Value of L is same as used in Soil Adjusted Vegetation Index (SAVI).

4) Soil \& Atmospherically Resistant Vegetation Index (SARVI): It is the ratio of NIR and RB bands, where RB means a combination of Red and Blue bands. This term can be calculated as follows.

SARVI $=\left(\rho_{\mathrm{NIR}}-\rho_{\mathrm{RB}}\right)(1+\mathrm{L}) /\left(\rho_{\mathrm{NIR}}+\rho_{\mathrm{RB}}+\mathrm{L}\right)[15](18)$ 


$$
\mathrm{RB}=\left(\rho_{\text {Red }}\right)-\gamma *\left(\rho_{\text {Red }}-\rho_{\text {Blue }}\right)
$$

where $\gamma=1$ and $\mathrm{L}$ is same as used in Soil Adjusted Vegetation Index (SAVI).

5) Green Normalized Vegetation Index (GNDVI): It is the ratio of the reflectance of NIR and Green bands.

$$
\operatorname{GrNDVI}=\frac{\left(\rho_{\mathrm{NIR}}-\rho_{\mathrm{Green}}\right)}{\left(\rho_{\mathrm{NIR}}+\rho_{\mathrm{Green}}\right)}[15]
$$

All Vegetation Indices are bounded in the range of -1 and +1 . For thick vegetation like the forest, it reaches +1 , for bare soil they are around 0 and for water bodies and snow/cloud it is negative.

For normal vegetation, is in the range of 0.3 to 0.7 . So almost all crop areas.

\section{RESULTS AND DISCUSSIONS}

In this paper, we compared proposed method with state of the art performance measurements like Standard Deviation (SD), Peak Signal to Noise Ratio (PSNR) and Structural Similarity Index (SSIM) with existing denoising and enhancement techniques i.e. Wavelet Filter based denoising enhancement (Wv),Histogram Equalization (HE) (Wv+HE) and Curvelet (C),Histogram Equalization (HE) $(\mathrm{C}+\mathrm{HE})$.Images are taken from Landsat 8 downloaded from USGS website covering both Kharif and Rabi seasons for the period 2015 to 2017.After extraction of Region of Interest (RoI), i.e., Chittoor region these algorithms are applied to test the quality of the image. Extracted Area of Interest (AOI) of Chittoor region from this imagery (months of January, March, August, and December for the years of 2015, 2016 and for 2017, from January to August).During these seasons lot of texture, variabilities were seen over in this area due to mountains, rivers, roads, and crops, etc. Initially, Additive Gaussian Noise with zero means $(\mu=0)$ with some variance $(\sigma=0,5,10,15,20)$ was added to the dataset and applied the proposed algorithm of denoising and enhancement. Datasets are selected that covers entire growth period of the plant from flowering to ripening state. Along with the enhancement the work has been extended to Vegetation indices study. Then the same was processed by denoising algorithms, wavelet-based and UDT-CWT based approach. By this study, almost all bands affected by noise are almost uniform and similar. Even though it is a very small variation that effect was studied and can be concluded that effect of noise is high in NIR, Red, and Green compared to the Blue band. Interestingly, this effect is inversely related to the wavelength of a particular band. Seasonally also can be concluded that during growing season rather than ripening state. Statistically, for the months of August (Image 3,7 ) and December (Image 4, 8) got very good performance (Even though most of the area of the pixel around the mean). For the remaining months because of low vegetation, the soil is the most dominating one in the scene. Obviously, most of the pixels are centred on and around Zero. On the other hand, the effect of noise is very high for NDVI compared to all other indices and SARVI, and GrNDVI shows better robust nature in noise attacks (which contains bands of Blue or Green in addition to NIR and Red bands). The reason is, NIR and Red bands are noisier effected than the green band (Same can be observed in the band reflectance graph).To avoid misleading while denoising of satellite imagery is more appreciated step, while the estimating of Vegetation indices and development of models based on various vegetation indices. This phenomenon is not uniform for the entire crop cycle. The Fig. (5) shows the images of noisy and denoised images. From Fig. 5(a), (b) and (c) it is clear that Information from this imagery is blurred and not supposed for analysis and process too. So this problem has been rectified using the proposed algorithm, which is represented in (d).

TABLE II

Performance Metrics for Proposed Method of Denoising And Enhancement

\begin{tabular}{|c|l|l|l|l|l|l|l|l|l|}
\hline \multirow{2}{*}{$\begin{array}{c}\text { Image } \\
\#\end{array}$} & \multicolumn{3}{|c|}{ Standard Deviation (SD) } & \multicolumn{3}{c|}{ PSNR (dB) } & \multicolumn{3}{c|}{ SSIM } \\
\cline { 2 - 11 } & Proposed & Cv+HE & Wv+HE & Proposed & Cv+HE & Wv+HE & Proposed & Cv+HE & Wv+HE \\
\hline 1 & $\mathbf{3 7}$ & 38 & 41 & $\mathbf{4 6}$ & 34 & 32 & $\mathbf{0 . 9 8}$ & 0.89 & 0.78 \\
\hline 2 & $\mathbf{5 1}$ & 54 & 58 & $\mathbf{3 5}$ & 31 & 28 & $\mathbf{0 . 9 7}$ & 0.92 & 0.83 \\
\hline 3 & $\mathbf{3 9}$ & 40 & 48 & $\mathbf{3 8}$ & 36 & 34 & $\mathbf{0 . 9 9}$ & 0.95 & 0.81 \\
\hline 4 & $\mathbf{2 1}$ & 23 & 29 & $\mathbf{4 4}$ & 40 & 39 & $\mathbf{1}$ & 0.91 & 0.75 \\
\hline 5 & $\mathbf{4 3}$ & 39 & 55 & $\mathbf{4 9}$ & 45 & 42 & $\mathbf{0 . 9 9}$ & 0.94 & 0.76 \\
\hline 6 & $\mathbf{4 9}$ & 43 & 58 & $\mathbf{4 1}$ & 36 & 32 & $\mathbf{0 . 9 7}$ & 0.9 & 0.77 \\
\hline 7 & $\mathbf{3 7}$ & 40 & 45 & $\mathbf{4 7}$ & 41 & 36 & $\mathbf{0 . 9 9}$ & 0.88 & 0.8 \\
\hline 8 & $\mathbf{3 6}$ & 39 & 42 & $\mathbf{4 4}$ & 40 & 36 & $\mathbf{0 . 9 9}$ & 0.86 & 0.84 \\
\hline 9 & $\mathbf{4 1}$ & 47 & 55 & $\mathbf{3 9}$ & 36 & 29 & $\mathbf{0 . 9 9}$ & 0.94 & 0.88 \\
\hline 10 & $\mathbf{4 8}$ & 50 & 59 & $\mathbf{4 0}$ & 39 & 37 & $\mathbf{0 . 9 8}$ & 0.97 & 0.91 \\
\hline
\end{tabular}

The below graph shows the relation between PSNR (of the Blue band) and Variance of the AWGN noise added in this paper. It shows the very sharp variation of the value between 5 and 10 and almost uniform from 10 to 30 with a small variation in the PSNR.The below Fig. 7 shows the variety of Standard deviations for available VIs and almost all indices show the same pattern except minor changes 


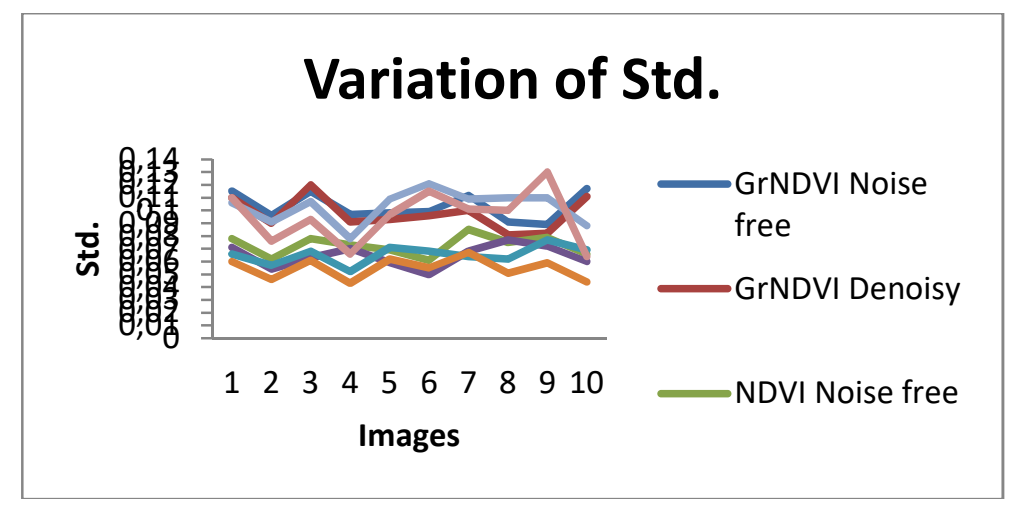

Fig. 7 Comparison of Variations of Standard Deviation for different VIs for denoisy and noise free images
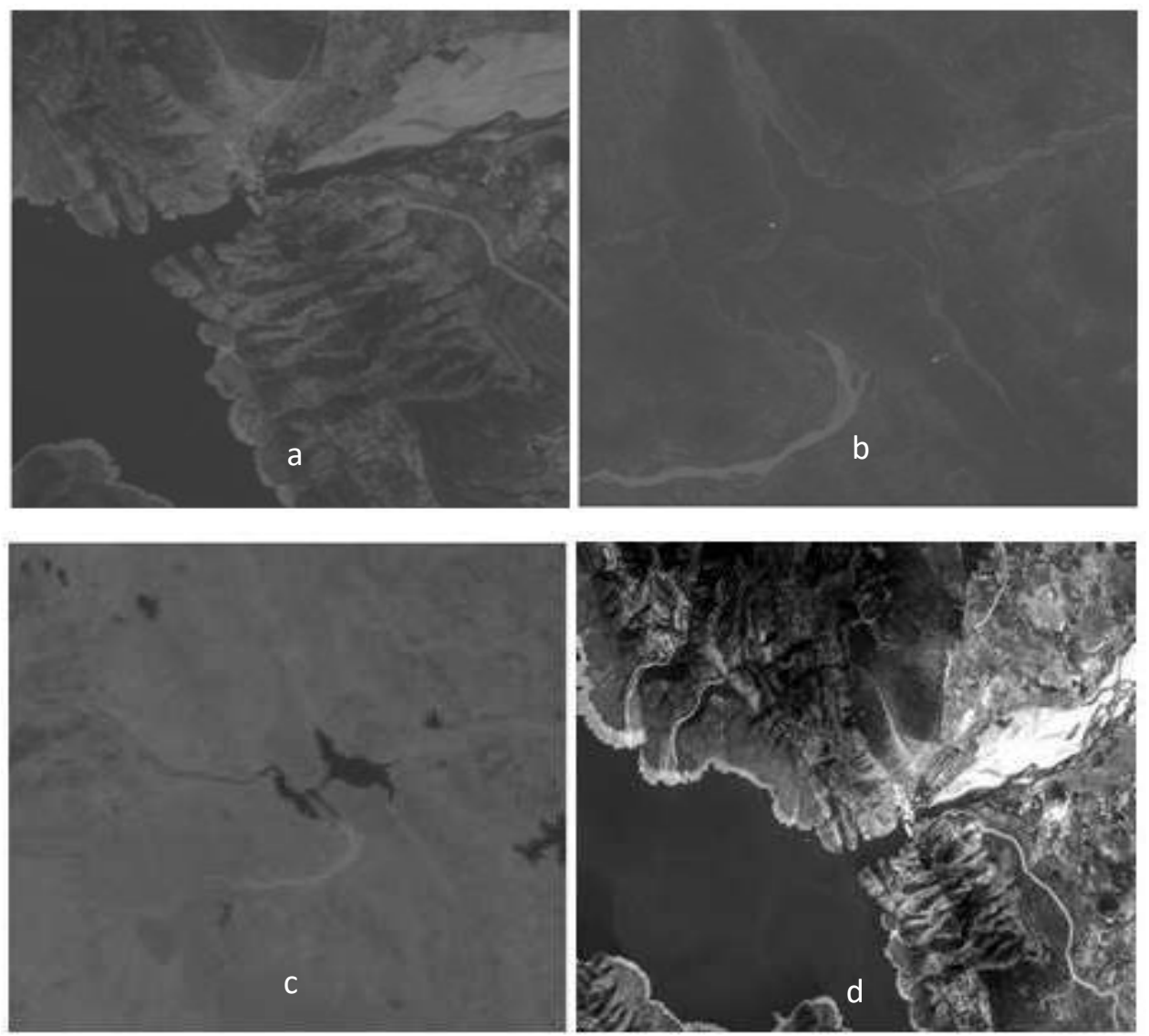

Fig. 5 Landsat8 Images for Chittoor region.(a) Noisy $(\sigma=5)$ (b) Noisy $(\sigma=10)$ (c) Noisy $(\sigma=20)$ (d) Denoised and enhanced image of the same scene

\section{CONCLUSION}

By this study,we can conclude that the proposed method enhances and denoised the image effectively in terms of SD, PSNR, and SSIM visually better than existing one. The attack of noise has been tested at various levels of plant stages starts from ripening to flowering. It denoises (AWGN) at each stage with some good numerical quantity at the output. Along with the enhancement the work has been extended to find Vegetation Indices (VI) and found the effect of noise on individual scene. It is more applicable to high-resolution images like Landsat, IRS satellites. So, noise should be removed (De-noise) before -processing by any means. This pre-processing stage must not disturb features of the received imagery. Noise is more severe in the growth stage of plant pixels compared with early state images So; denoising is more preferable to calculate VI rather than calculating on direct images. Wrong values in statistics, results in mediocre outcomes in crop yield prediction. In this study, images are tested thoroughly by covering all stages of vegetation pixels. For better accuracy and precision considered several VIs instead of only one. It is one of the few papers in this direction. Statistically, it has been proved that Quadtree wavelet-based method is better over Curvelet method. Even though the limits of VIs narrow $[0,1]$ there is a lot of scopes to mislead entire picture of a forest or crop estimation. A limited set of temporal data is one of the main challenges in Landsat imagery. So, out of all these indices, MSAVI and SARVI are more robust to the noise variations and maintains good performance. 


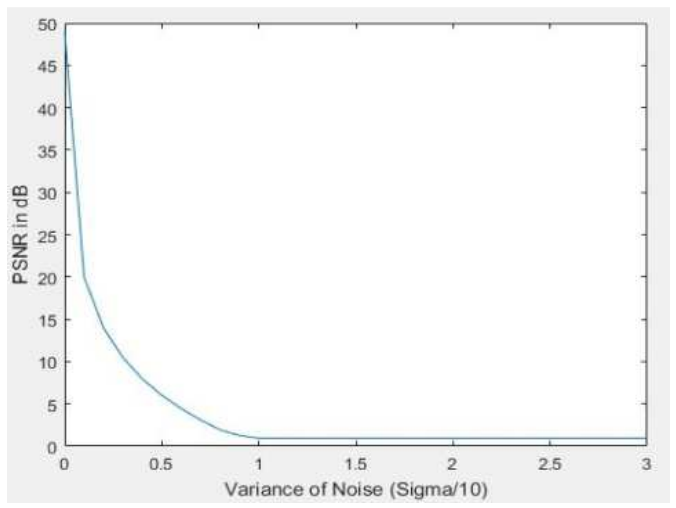

Fig. 6 Variations of PSNR with reference to Variance of the noise added

\section{REFERENCES}

[1] H. Demirel, S. Izadpanahi and G. Anbarjafari, 2009,Improved motion-based localized super resolution technique using discrete wavelet transform for low resolution video enhancement, 17th European Signal Processing Conference (EUSIPCO-2009), Edinburgh, Scotland, pp. 1097-1101.

[2] P. Rasti, I. Lusi, H. Demirel, R. Kiefer, and G. Anbarjafari,2014, Wavelet transform based new interpolation technique for satellite image resolution enhancement, IEEE International Conference on Aerospace Electronics and Remote Sensing Technology, pp. 185 188.

[3] Y. Piao, 1. Shin, and H. W. Park, "Image resolution enhancement using inter-subband correlation in wavelet domain", IEEE International Conference on Image Processing, 2007, Vol. 1, pp. I 445 - I - 448

[4] H.Tasmaz,H.Demirel, and G.Anbarjafari,2012,Satellite image enhancement by using dual tree complex wavelet transform: Denoising and illumination enhancement,20th IEEE Signal
Processing and Communications Applications Conferences(SIU2012),pp.1-4.

[5] Pejman Rasti1, Haci Taşmaz et. al., 2016, Satellite Image Enhancement: Systematic Approach For Denoising And Resolution, Vol. 91, Issue 3.

[6] D. L. Donoho, 1993,Nonlinear wavelet methods for recovery of signals, densities, and spectra from indirect and noisy data, in Proceedings of Symposia in Applied Mathematics. Vol 47.

[7] N.G.Kingsbury,1998,The dual-tree complex wave transforms: a new technique for shift variance and directional filters,IEEE Digital Signal Processing Workshop,pp.319-322.

[8] A. Green, M. Berman, P. Switzer, and M. Craig, 1988,A transformation for ordering multispectral data in terms of image quality with implications for noise removal, IEEE Transactions on Geoscience and Remote sensing,26,1, pp. 65-74.

[9] G. Chang, B. Yu, and M. Vetterli,2000,Adaptive wavelet thresholding for image denoising and compression, IEEE Transactions on Image Processing 9:(9), pp. 1532-1546.

[10] Wenli Liu, Peng He, Hui Li, Hongbo Yu,2012, Improvement on the Algorithm of Homomorphic Filtering, International Conference on Electrical and Computer Engineering, Vol. 11, pp. 120 - 124.

[11] I Wayan Nuarsa, F Nishio and Chiharu Hongo," Rice Yield Estimation Using Landsat ETM+ Data and Field Observation", Journal of Agricultural Science, Vol. 4, No. 3; pp 45-56, 2012.

[12] M. Akbari , A. r. Mamanpoush , A. Gieske, M. Miranzadeh , M. Torabi \& H. R. Salemi,2006, Crop and land cover classification in Iran using Landsat 7 imagery", International Journal of Remote Sensing, Vol. 27, No. 19, pp-4117-4135

[13] Kun Jia, Shunlin Liang, Xiangqin Wei, Yunjun Yao, Yingru Su, Bo Jiang and Xiaoxia Wang, 2014,Land Cover Classification of Landsat Data with Phenological Features Extracted from Time Series MODIS NDVI Data, Remote sensing, 6, pp-11518-11532.

[14] Thomas, V. Benning, and N. Ching, 1987, Classification of remotely sensed images, Adam Hilger, Bristol.

[15] Weicheng Wu," The Generalized Difference Vegetation Index (GDVI) for Dryland Characterization", Remote Sens. 2014, 6, pp1211-1233. 